\title{
Qui « décroche » de l'université ? Le cas des étudiants de la région Aquitaine (France)
}

\author{
Joël ZAFFRAN ${ }^{a^{*}} \&$ Maud AIGLE ${ }^{a}$ \\ ${ }^{a}$ Université de Bordeaux, Centre Émile Durkheim, France \\ * joel.zaffran@u-bordeaux.fr
}

\section{Résumé}

La lutte contre le décrochage à l'université recueille un large consensus auprès des différents acteurs politiques et universitaires. Quels que soient les référentiels, la nécessité d'un changement des conditions d'accompagnement des étudiants est souvent rappelée, avec à l'appui le taux de réussite en fin de 1 re année de licence qui reste insuffisant et les abandons sont en nombre importants. Or, la manière de présenter publiquement le problème mélange les situations d'abandon définitif des études, de réorientation et d'interruption provisoire des études. Qui décroche réellement à l'université et combien sont-ils vraiment? Notre communication a deux objectifs. Le premier objectif est de présenter les résultats d'une vaste enquête par questionnaire menée à l'échelle d'une région auprès des sortants sans diplôme. Au regard de ces résultats, le second objectif est de pointer les facteurs explicatifs du décrochage à l'université mais surtout de souligner la nature processuelle du problème.

Mots clés : université, décrochage, disqualification publique, inégalités

\section{Who dropout from the university? The case of students from the Aquitaine region (France)}

\begin{abstract}
The fight against dropping out of university has garnered a broad consensus among the various political and academic players. Whatever the benchmarks, the need for a change in the conditions of support for students is often recalled,
\end{abstract}


supported by the success rate at the end of the 1 st year of the license which remains insufficient and the number of dropouts is significant. However, the way of presenting the problem publicly mixes up the situations of definitive abandonment of studies, reorientation and temporary interruption of studies. Who is really dropping out of college and how many are there really? Our communication has two objectives. The first objective is to present the results of a large questionnaire survey carried out at the regional level among those leaving without a diploma. In view of these results, the second objective is to point out the explanatory factors for dropping out of university but above all to underline the processual nature of the problem.

Keywords: university, dropout, public disqualification, inequalities

\section{Introduction}

Longtemps perçu comme acceptable, voire comme un gage d'excellence du diplôme délivré (Pérennès et Pinte, 2012), le « décrochage à l'université » est devenu insupportable. Il est devenu un problème dont s'emparent des claims-makers qui mélangent les fausses prédictions et les mauvais chiffres mais qui définissent le guide à l'action publique. Un écart existe entre la publicisation du problème et sa nature que les données disponibles, quantitatives et qualitatives, aident à connaître. Sur le plan quantitatif, on sait que le fait d'être titulaire d'un baccalauréat professionnel, d'appartenir à un milieu modeste ou d'être primo-accédant au baccalauréat dans sa famille accroît le risque d'abandonner ses études universitaires en première année (Lemaire, 2005 ; Menard, 2017). Sur le plan qualitatif, les profils de « décrocheurs » s'organisent généralement autour des deux dimensions structurantes des parcours d'études : le rapport de l'étudiant à la formation initiale et son rapport au marché de l'emploi (Beaupère et Boudesseul, 2009). Ce que l'on ne sait pas encore tout à fait est le nombre exact de « décrocheurs ». Le connaître est une manière de vérifier su leur nombre est aussi élevé que veulent faire accroire les claims-makers. C'est l'objet de 
notre communication. Considérant que la publicisation d'un problème ne dépend en aucun cas de sa nature intrinsèque mais des représentations portées par les acteurs, on présente les résultats d'une vaste enquête par questionnaire menée en France à l'échelle de l'Aquitaine auprès des étudiants en général et des décrocheurs en particulier. L'enquête permet trois choses : elle relativise l'ampleur du problème ; elle pointe les facteurs explicatifs du décrochage à l'université ; elle insiste sur la dimension processuelle du phénomène.

\section{Qui part et qui revient : suivre les étudiants}

Le débat public et les textes réglementaires, nationaux ou supranationaux, auxquels donne lieu le « décrochage à l'université » témoignent d'un souci de faire réussir les jeunes et combattre l'échec à l'université. Or, ce qui pourrait être vu comme un échec ne l'est pas toujours. Ainsi, être à l'université peut être une stratégie d'attente des jeunes qui voient le premier cycle universitaire comme l'antichambre d'une future formation hors d'un cadre universitaire au sein duquel il est possible de s'épanouir autrement. Les étudiants qui échouent aux examens ne peuvent pas être mis sur le même plan que les étudiants qui quittent l'université sans diplôme pour s'inscrire dans l'école de leur choix (Ménard, 2017). De même, ce qui est vu comme un abandon des études pourrait n'être qu'une parenthèse dans le parcours du jeune. Celui-ci peut interrompre ses études une année et les reprendre, sous la même forme ou pas, l'année suivante. D'une certaine façon, les étudiants qui sortent de l'université sans diplôme ne cessent pas d'être des étudiants pour autant puisque le retour en formation est possible.

Le taux élevé de sortants non diplômés de l'université, accompagné d'une dramatisation de l'échec, notamment en L1 avec près de $60 \%$ d'échec, mérite d'être revue à l'aune des parcours des étudiants. Les données du système d'information sur le suivi des étudiants (SISE) du ministère de l'Éducation nationale ouvrent une première 
piste $^{1}$. Elles montrent que sur les 165987 néo-bacheliers inscrits en L1 en 2015, 41,6 \% accèdent en L2 l'année suivante. Elles montrent aussi que les $60 \%$ d'échec sont l'agrégation du redoublement (28,3\% des néo-bacheliers redoublent leur L1), d'un changement d'orientation (2,3\%) et de sortie de l'université $(27,8 \%)$. A cela s'ajoute un taux de passage en L2 qui dépend de l'origine sociale. Tandis que 51,3\% des étudiants « très favorisés » (au sens du SISE) accèdent au niveau supérieur l'année suivante, ils ne sont que $32,5 \%$ parmi les étudiants considérés comme « défavorisés ». Si les taux d'échec font problème, la nature socialement déterminée de l'échec devrait l'être aussi puisque les étudiants n'ont pas la même probabilité de réussir ou d'échouer selon leur origine sociale (Romainville, Michaut, 2012). Outre l'origine sociale, le type de baccalauréat a un effet sur les parcours postbac.

Dans une étude portant sur le panel de bacheliers ayant obtenu leur baccalauréat en 2002 (panel entré en $6^{\text {ème }}$ en 1995), Lemaire (2005) met en évidence les caractéristiques spécifiques des bacheliers ne poursuivant pas leurs études dans l'enseignement supérieur. Les deux tiers sont titulaires d'un baccalauréat professionnel, et une large majorité accuse un retard dans leur parcours scolaire. L'exploitation des enquêtes Génération du Céreq met aussi en évidence l'impact du parcours scolaire sur la sortie sans diplôme de l'enseignement supérieur. Un redoublement avant l'entrée dans le supérieur expose au risque de sortie sans diplôme (Gury et Moullet, 2007 ; Calmand, Ménard et Mora, 2015). Enfin, les motifs de sortie évoqués par les sortants non diplômés de l'enseignement supérieur sont, outre les choix d'orientation et les problèmes de santé, les conditions de vie. L'abandon précoce peut avoir des causes 
financières ou être lié à une activité concurrente menée en parallèle des études (Galland et Houzel, 2009 ; Observatoire de la vie étudiante, 2016).

Cependant, et comme on vient de l'indiquer, parmi les néo-bacheliers ayant quitté l'université, tous n'ont pas abandonné les études. Certains ont pu s'inscrire dans une formation paramédicale et sociale, une école de commerce ou une formation relevant d'une autre tutelle ministérielle que l'Enseignement supérieur. Or, le SISE ne permet pas d'identifier ces parcours. On ne sait donc pas avec précision parmi les étudiants qui abandonnent leurs études qui sont véritablement les étudiants qui quittent définitivement l'enseignement supérieur et ceux qui y retournent immédiatement après en être sortis ${ }^{2}$. Cette méconnaissance est d'autant plus problématique que l'attribution des dotations financières aux universités se fonde, entre autres critères, sur le taux de réussite en licence. Depuis 2009, le SYMPA (Système de répartition des moyens à la performance et à l'activité) indexe l'attribution des crédits aux universités à leurs résultats pédagogiques et académiques (Aschieri, 2012). Outre le nombre de masters délivrés et la qualité des unités de recherche, le taux de réussite en licence sert au calcul de la dotation des établissements. De sorte que c'est la rétention des effectifs dans les filières qu'il importe de parfaire plutôt que la sécurisation du parcours de l'étudiant sortant (Millet, 2012 ; Bodin et Orange, 2013), alors même que parmi les $60 \%$ d'étudiants considérés en échec, peu d'entre eux ne poursuivent pas leurs études l'année suivante.

Dans ce cas, qui sont les « abandonnistes » et comment se poursuit leur parcours d'études ?

${ }^{2} \mathrm{Ce}$ critère d'immédiateté permet de ne pas inclure les adultes en reprise d'études, qui renvoient à une autre catégorie d'étudiants. 
Alors que le «problème du décrochage universitaire » se cristallise sur l'échec et la sortie sans diplôme, cette interrogation articule deux aspects du phénomène, en l'occurrence le départ et le retour aux études universitaires, y compris dans un établissement non recensé par le ministère de l'enseignement supérieur. Elle repose sur l'hypothèse d'une cohérence biographique derrière les changements dans le parcours de formation. Bien que notre approche soit quantitative, cette hypothèse s'inspire des travaux qui déconstruisent la réussite en introduisant l'idée que les bacheliers, toujours plus nombreux à s'inscrire à l'université, ne poursuivent pas tous les mêmes buts et ne sont pas réductibles à un profil. Alors que l'association de la réussite au diplôme tend à figer l'indicateur des sortants non diplômés, les logiques qui sous-tendent l'expérience étudiante le nuancent fortement. Ainsi, la typologie construite par Beaupère et Boudesseul (2009) à partir du rapport de l'étudiant à la formation initiale et de son rapport au marché de l'emploi présente quatre profils de décrocheurs. La plus ou moins grande importance accordée au diplôme croisée avec le degré d'anticipation de l'insertion professionnelle peut conduire l'étudiant à sortir de l'université sans qu'il envisage son départ comme un problème. L'abandon est plutôt vu comme un moyen de parvenir à une fin différente des finalités propres à l'université.

L'obtention du diplôme au sein de l'établissement d'inscription n'est pas pour eux une priorité, soit qu'ils cherchent à s'insérer rapidement dans la vie active soit qu'ils envisagent une réorientation vers une autre formation. Les étudiants qui établissent un lien fort entre la réussite académique et l'insertion professionnelle n'envisagent pas leur abandon comme une rupture définitive. Au contraire, ils ont un objectif de retour rapide en formation soutenu par leur croyance forte en la valeur du diplôme comme sésame sur le marché du travail. Le « décrochage » renvoie aussi à une démobilisation dont la source est la tension entre le projet d'avenir, le projet 
d'apprendre et la socialisation. L'enquête menée par Jellab (2011) auprès d'étudiants en L1 montre bien qu'il suffit que la sociabilité l'emporte sur l'investissement scolaire ou que le projet professionnel apparaisse en décalage avec les études poursuivies pour que le processus d'abandon se mette en œuvre. Par ailleurs, l'inscription à l'université ne relève pas seulement d'une démarche visant à obtenir un diplôme mais procède aussi d'une stratégie visant à se libérer pour un temps des contraintes sociales (David, Melnik-Olive, 2014) et, comme cela est plus particulièrement le cas des bacheliers professionnels, se soustraire aux exigences du monde professionnel en différant l'entrée sur le marché du travail pour goûter à un monde dont l'accès leur semblait interdit (Beaud et Pialoux, 2001).

Pour ces raisons, l'analyse doit prendre en compte le parcours d'accès, de sortie et de retour aux études supérieures. Sur un plan méthodologique, il s'agira de compléter les nombreuses approches qualitatives par une approche quantitative des facteurs explicatifs de l'abandon des études universitaires et du retour en formation des «abandonnistes ». On distingue donc parmi les étudiants qui sortent non diplômés de l'enseignement supérieur ceux qui y retournent dans un délai inférieur à 24 mois $^{3}$ de ceux qui n'y retournent pas après avoir passé une année au moins en dehors de tout système de formation. Le terrain est constitué par les trois universités membres de la ComUE d'Aquitaine, ce qui permet de porter l'analyse du devenir de 56046 étudiants inscrits en 2015-2016 dans l'une d'elles. Le choix est fait de suivre les parcours en licence et en master puisque l'abandon précoce évoque l'idée d'une sortie des études sans obtenir le diplôme visé au moment de l'inscription, quand bien même les

${ }^{3}$ Bien qu'arbitraire, ce délai englobe les réorientations après le premier semestre et les réinscriptions après deux semestres. 
conséquences de la sortie sont sans doute différentes selon le niveau (L1, L2 ou L3) et la nature du diplôme (licence ou master). Par ailleurs, l'analyse préalable des données administratives SISE laissait entrevoir une forte propension des inscrits en cursus master à interrompre leur formation avant l'obtention du diplôme préparé. Ce premier constat a conforté le choix d'inclure les masters dans l'analyse.

\section{Encart méthodologique}

Le champ d'étude a été défini comme l'ensemble des inscrits à une année N, n'ayant pas obtenu le diplôme préparé et ne s'étant pas réinscrit l'année N+1. Cette définition exclut du champ d'observation une large partie des interruptions d'études en cours de formation, à savoir toutes les interruptions ne se soldant pas l'année suivante par une sortie du système éducatif. De sorte que l'échantillon ne retient pas les étudiants qui redoublent ou se réorientent mais ceux qui abandonnent leurs études sans les reprendre, d'une manière ou d'une autre, l'année suivante.

Par ailleurs, l'enquête permet de connaître le devenir des étudiants à N+1 (année 2016-2017) et en N+2 (année 2017-2018). Ainsi, nous pouvons estimer la part de sortant en N+1 parmi l'ensemble des inscrits mais également la part des reprises d'étude en N+2 parmi l'ensemble des sortants. Pour le dire autrement, l'échantillon concerne les sorties « sèches » de l'université ( $c f$. Figure 1).

Figure 1. Schéma du dispositif de l'enquête Sortants. 


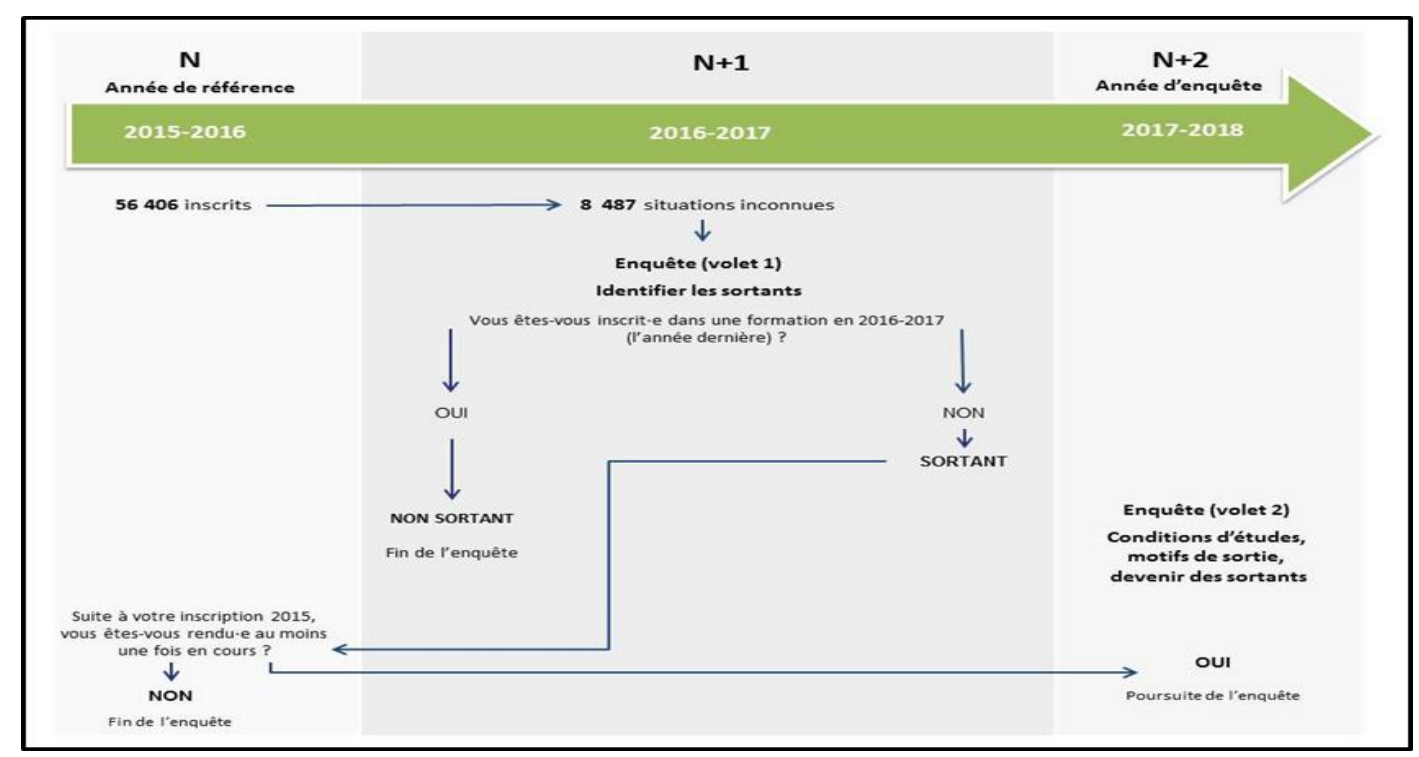

L'échantillon est construit par appariement de trois fichiers administratifs : les données Apogée et SISE des trois universités membres de la ComUE d'Aquitaine (l'université de Bordeaux, l'université de Bordeaux-Montaigne et l'université de Pau et des Pays de l'Adour ainsi que les données SCOLARITE (soit les inscrits de BTS et CPGE) du Rectorat. L'appariement a permis d'identifier les étudiants inscrits en 20152016 qui sont non diplômés à l'issue de cette même année4 et non retrouvés l'année suivante dans les fichiers SISE et SCOLARITÉ. En conséquence de quoi ce sont 8487 étudiants inscrits en 2015-2016 en cursus DUT, licence et master, non diplômés et non retrouvés dans les fichiers Apogée, SISE et SCOLARITÉ l'année suivante qui furent interrogés de septembre à décembre 2017. Parmi eux, 4918 ont répondu à l'enquête,

${ }^{4}$ Par non diplômé, nous entendons tous les étudiants n'ayant pas obtenu le diplôme préparé dans le cadre du cursus d'inscription. Cela comprend donc les étudiants inscrits en master diplômés du premier cycle mais n'ayant pas obtenu un titre de second cycle. Cela comprend également les étudiants de licence 1, licence $Q$ et DUT 1 ayant validé leur année mais n'ayant pas obtenu le diplôme du cycle. 
soit un taux de retour de 58\%. Ce taux est très satisfaisant compte tenu de la difficulté à approcher ce public.

Cela a été possible grâce à des relances téléphoniques et une équipe dédiée au sein de chaque observatoire des parcours étudiants de chaque université. Au moment de lancer l'enquête, tous les établissements membres de la ComUE ont été sollicités. Une partie seulement des établissements ont accepté de s'inscrire dans la démarche, en l'occurrence trois universités. Les deux écoles d'ingénieurs ainsi que l'IEP n'ont pas souhaité intégrer le dispositif, la question du décrochage ne se présentant pas selon eux comme une préoccupation forte au sein de leur établissement. Ce refus est une première limite au dispositif d'enquête puisqu'il restreint le champ d'observation au seul cadre universitaire, et qu'il ne permet pas de retracer le parcours des étudiants dans les établissements sélectifs.

Avant de s'attacher à identifier les trajectoires étudiantes, il convient de décrire les caractéristiques de notre échantillon. En 2015-2016, 56406 étudiants sont inscrits dans les trois universités membres de la ComUE d'Aquitaine, en cursus DUT, licence et master. Les filles y sont plus nombreuses (57,5\% des inscrits) et un tiers des inscrits (33\%) est enfant de cadres ou de professions intellectuelles supérieures tandis que $17 \%$ sont des enfants d'employés et $10 \%$ enfants d'ouvriers. Outre l'inégale répartition des différentes catégories sociales parmi les inscrits, on note que les catégories les plus représentées sur les bancs de l'université sont encore aujourd'hui5 les moins présentes

${ }^{5}$ Pierre Bourdieu et Jean-Claude Passeron formulaient déjà ce constat en 1964 dans Les héritiers. À titre de rappel, les cadres et professions intellectuelles supérieures représentent 9,2\% de la population active (source : Insee, RP2015 exploitation complémentaire, géographie au 01/01/2017). 10 
dans la population active. Par ailleurs, il s'agit d'une population relativement jeune puisque trois étudiants sur quatre ont moins de 25 ans et seuls 5,6\% des inscrits sont âgés de 30 ans et plus. Enfin, $66 \%$ des inscrits ont obtenu leur baccalauréat dans l'académie de Bordeaux et $7 \%$ sont de nationalité étrangère (tableau 1).

Tableau1 : Caractéristiques sociodémographiques des inscrits en 2015-2016 et répartition par cursus.

\begin{tabular}{|c|c|c|c|c|c|c|c|}
\hline & & & & Répartit & on par cu & us en $\%$ & \\
\hline $\begin{array}{l}\text { *\% calculés } \\
\text { observation }\end{array}$ & $\begin{array}{l}\text { partir des } \\
\text { valides }\end{array}$ & Effectifs & $\begin{array}{l}\% \text { dans la } \\
\text { population* }\end{array}$ & $\begin{array}{l}\text { Cursus } \\
\text { licence }\end{array}$ & $\begin{array}{l}\text { Cursus } \\
\text { master }\end{array}$ & $\begin{array}{l}\text { Cursus } \\
\text { DUT }\end{array}$ & CPGE \\
\hline Ensemble d & inscrits & 56406 & $100 \%$ & $63,4 \%$ & $25,2 \%$ & $9,1 \%$ & $2,3 \%$ \\
\hline & Hommes & 24000 & $42,5 \%$ & $61,2 \%$ & $23,8 \%$ & $12,2 \%$ & $2,7 \%$ \\
\hline & Femmes & 32406 & $57,5 \%$ & $65 \%$ & $26,2 \%$ & $6,7 \%$ & $2 \%$ \\
\hline & Français & 52469 & $93 \%$ & $64 \%$ & $24,1 \%$ & $9,5 \%$ & $2,4 \%$ \\
\hline & Étrangers & 3937 & $7 \%$ & $55,5 \%$ & $39,8 \%$ & $3,9 \%$ & $0,8 \%$ \\
\hline & $\begin{array}{l}\text { Agriculteur } \\
\text { exploitant }\end{array}$ & 1216 & $2,3 \%$ & $60,2 \%$ & $26,3 \%$ & $10,9 \%$ & $2,5 \%$ \\
\hline PCS & $\begin{array}{l}\text { Artisan, } \\
\text { comme, chef } \\
\text { d'entr. }\end{array}$ & 5007 & $9,5 \%$ & $65,7 \%$ & $22,4 \%$ & $9 \%$ & $2,9 \%$ \\
\hline
\end{tabular}


The Journal of Quality in Education (JoQiE) Vol.11, Nº17, May 2021

\begin{tabular}{|c|c|c|c|c|c|c|c|}
\hline & $\begin{array}{l}\text { Cadre et prof. } \\
\text { int. sup. }\end{array}$ & 17317 & $33 \%$ & $62,3 \%$ & $23,8 \%$ & $10 \%$ & $3,8 \%$ \\
\hline & $\begin{array}{l}\text { Prof. } \\
\text { intermédiaire }\end{array}$ & 8239 & $15,7 \%$ & $66,4 \%$ & $21,1 \%$ & $10,4 \%$ & $2,1 \%$ \\
\hline & Employé & 8990 & $17,1 \%$ & $68,8 \%$ & $19,5 \%$ & $10,2 \%$ & $1,5 \%$ \\
\hline & Ouvrier & 5123 & $9,8 \%$ & $68,5 \%$ & $21,6 \%$ & $8,8 \%$ & $1,1 \%$ \\
\hline & Inactif & 2924 & $5,6 \%$ & $70,1 \%$ & $22,2 \%$ & $6,8 \%$ & $0,9 \%$ \\
\hline & Retraité & 3726 & $7,1 \%$ & $53,4 \%$ & $39,7 \%$ & $5,6 \%$ & $1,3 \%$ \\
\hline & Non renseigné & 3864 & - & - & - & - & - \\
\hline \multirow{5}{*}{$\begin{array}{l}\text { Académie } \\
\text { d'obtention } \\
\text { du } \\
\text { baccalauréat }\end{array}$} & Bordeaux & 36737 & $65,8 \%$ & $69,0 \%$ & $18,5 \%$ & $10,0 \%$ & $2,5 \%$ \\
\hline & $\begin{array}{l}\text { France métro. } \\
\text { (Hors } \\
\text { Bordeaux) }\end{array}$ & 14431 & $25,8 \%$ & $51,5 \%$ & $37,4 \%$ & $8,8 \%$ & $2,3 \%$ \\
\hline & DROM COM & 1996 & $3,6 \%$ & $66,3 \%$ & $27,8 \%$ & $4,5 \%$ & $1,5 \%$ \\
\hline & Étranger & 2709 & $4,8 \%$ & $51,3 \%$ & $44,3 \%$ & $3,8 \%$ & $0,7 \%$ \\
\hline & Non renseigné & 533 & - & - & - & - & - \\
\hline \multirow{4}{*}{$\begin{array}{l}\text { Type de } \\
\text { Baccalauréa } \\
\text { t }\end{array}$} & Général & 45949 & $81,5 \%$ & $63,6 \%$ & $25,6 \%$ & $8,1 \%$ & $2,7 \%$ \\
\hline & Technologique & 6291 & $11,2 \%$ & $62,7 \%$ & $17,3 \%$ & $19,2 \%$ & $0,8 \%$ \\
\hline & Professionnel & 1784 & $3,2 \%$ & $85,7 \%$ & $7,7 \%$ & $6,7 \%$ & - \\
\hline & Dispense & 2382 & $4,2 \%$ & $45,7 \%$ & $51,2 \%$ & $3 \%$ & - \\
\hline
\end{tabular}

Sources : SISE 2015-2016. 
Lecture :parmi les 56406 étudiants inscrits en 2015-2016 dans l'une des trois universités de la ComUE, 63,4\% sont en cursus Licence

\section{Parcours d'étudiants et causes de l'abandon}

Le tableau 1 atteste de l'hétérogénéité des publics à l'université. Celle-ci est souvent présentée comme la conséquence directe de la massification de l'accès à l'enseignement supérieur. Cependant, une lecture du tableau 1 par niveaux et filières fait remarquer que la répartition des inscrits n'est pas indépendante de trois facteurs. En premier lieu, l'orientation genrée des publics est particulièrement marquée. Les femmes sont surreprésentées en licence et en master alors qu'elles sont sous-représentées dans les cursus DUT et CPGE. En outre, d'importants écarts sont à noter entre discipline. Tandis que les femmes sont nombreuses en lettres et sciences humaines, elles le sont nettement en sciences fondamentales et appliquée. En deuxième lieu, les enfants de cadres et des professions intellectuelles supérieurs sont très majoritaires parmi le public étudiants (33\%) et parmi les élèves de CPEG (3,8 \%). En troisième lieu, l'origine scolaire permet de pointer une autre différence remarquable : $81,5 \%$ des inscrits sont titulaires d'un baccalauréat général alors qu'ils ne sont que 3,2 \% diplômés d'un baccalauréat professionnel. Considérés ensemble, ces facteurs nuancent l'image d'une université que la massification aurait diversifiée. Le tableau 1 rappelle que l'université est un espace sélectif et hiérarchisé.

Les données de l'enquête permettent de connaître le devenir des étudiants en 2016-2017 . Leur parcours décrit plusieurs situations : une poursuite d'études, un

${ }^{6}$ Pour des raisons de suivi et d'attrition, l'effectif total passe alors à 52839 individus soit une seconde base qui représente $94 \%$ de la base initiale. 
redoublement, une réorientation, une sortie diplômée, une sortie avant l'obtention du diplôme préparé lors de l'inscription en 2015-2016 (tableau 2).

Tableau 2 : Devenir en 2016-2017 des étudiants inscrits en 2015-2016

\begin{tabular}{|l|l|l|}
\cline { 2 - 3 } \multicolumn{1}{l|}{} & Effectifs & $\%$ \\
\hline Poursuite d'étude & 26384 & 49,9 \\
Redoublement & 9754 & 18,5 \\
Réorientation & 6058 & 11,5 \\
Sortie diplômée & 7933 & 15 \\
Sortie sans diplôme & 2710 & 5,1 \\
Total & 52839 & 100 \\
\hline
\end{tabular}

Sources : SISE 2015-2016, SISE 2016-2017, Enquête sortants (ComUE d'Aquitaine)

Lecture : $5.1 \%$ des étudiants inscrits en 2015-2016 dans l'un des trois universités de la ComUE sont en sortie sans diplôme en 2016-2017.

Parmi les étudiants dont la situation en 2016-2017 est connue, la moitié sont réinscrits dans une formation de niveau supérieur. Parmi eux, $12 \%$ ont changé de champ disciplinaire une année après leur première inscription ${ }^{7}$. Les étudiants réinscrits dans une formation de niveau identique à celle suivie en 2015-2016 sont des «redoublants ». Ils représentent $18,5 \%$ des situations connues, et certains ne se sont pas réinscrits dans la même filière : $14 \%$ ont changé de discipline. La « réorientation » concerne les étudiants réinscrits dans une formation de niveau inférieur à celle suivie en 2015-2016 et les étudiants réinscrits dans une formation conduisant à un autre type de

${ }^{7}$ Le secteur disciplinaire est la variable la plus agrégée de la nomenclature SISE. Elle comporte 7 disciplines : Sciences, Staps, Droit, Économie-Aes, Iut secondaire, Iut tertiaire, Lettres, Sciences humaines, Santé. 
diplôme (BTS, écoles sélectives). Elle concerne 11,5\% des inscrits ${ }^{8}$. Les étudiants

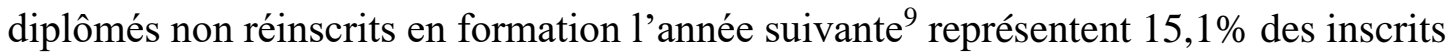
de 2015-2016. Au regard de notre objet, la dernière situation est la plus intéressante : parmi l'ensemble des inscrits en 2015-2016, peu ont quitté l'université avant d'avoir obtenu le diplôme associé à leur inscription. Les abandons «purs » ne représentent que 5,1\% de l'ensemble des inscrits, soit 2710 individus. Par ailleurs, l'abandon ne va pas de pair obligatoirement avec une non admission aux examens puisque sur 100 sorties non diplômée, 12 environ interviennent malgré une admission aux examens ${ }^{10}$ (graphique 1).

${ }^{8}$ Parmi eux, $21 \%$ se sont inscrits dans une école sélective (IEP, ingénieur, commerce), $18 \%$ en BTS, $16 \%$ dans une année de préparation aux concours, $13 \%$ dans une formation LMD de niveau inférieur au niveau atteint, $8 \%$ dans une école du secteur social ou paramédical, $5 \%$ en DUT, $4 \%$ dans une formation professionnelle et un peu moins de $2 \%$ dans une formation de l'enseignement secondaire.

${ }^{9}$ Cette catégorie regroupe les étudiants diplômés en 2015-2016, non retrouvés dans les fichiers SISE l'année suivante. Cela ne signifie pas que tous auraient quitté le système de formation mais que certains auraient poursuivi leurs étules dans des établissements non recensés par le SISE. Dans la mesure où l'enquête porte principalement sur les sortants non-diplômés, le devenir des sortants diplômés est hors champ. Il se peut donc que la part des sortants diplômés soit surévaluée au regard de la part des poursuites d'études et des réorientations.

${ }^{10}$ On voit que $7 \%$ environ des redoublants sont des admis. Cela s'explique par la stratégie d'étudiants visant des masters sélectifs (comme c'est le cas en psychologie) et qui préfèrent redoubler malgré leur admission aux examens pour présenter un meilleur dossier l'année suivante. Par ailleurs, 4 \% des sortants diplômés n'ont pas été admis aux examens, mais ils 
Graphique 1 : Trajectoires en 2016-2017 des inscrits en 2015-2016 selon leur réussite aux examens

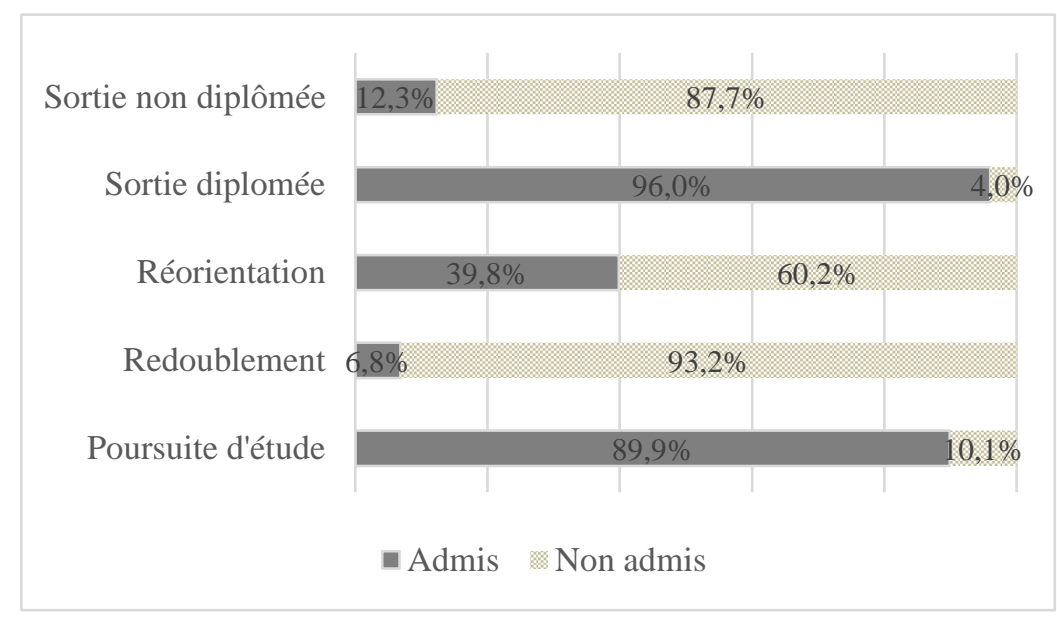

Source : SISE 2015-2016, SISE 2016-2017. Champ : inscrits 2015-2016 des trois universités membres de la ComUE d'Aquitaine, dut, licence, master (52 839 observations valides)

Lecture : Sur 100 admis à l'issue des épreuves d'examen de la filière d'inscription, 90 poursuivent leurs études dans cette filière.

De façon logique, les étudiants admis sont surreprésentés parmi ceux engagés dans une poursuite d'études tandis que les non admis ${ }^{11}$ sont surreprésentés parmi les redoublements, les réorientations et les sorties anticipées. Toutefois, des comportements atypiques invitent à nuancer cette relation. En effet, $10 \%$ des étudiants n'ayant pas validé leurs examens poursuivent leurs études l'année suivante dans un niveau supérieur tandis que $39 \%$ des admis se réorientent, que $7 \%$ environ redoublent et surtout que

sont titulaires d'un autre diplôme. C'est le cas des étudiants d'une licence professionnelle par exemple qui échouent leur master.

${ }^{11}$ Il est à noter qu'être non admis n'est imputable directement à un échec, certains étudiants ayant décidé de ne pas passer tout ou une partie de leurs examens pour différentes raisons qu'il n'a pas été possible de connaître. 
$12 \%$ des étudiants qui interrompent leurs études avant l'obtention du diplômé préparé ont été admis à leurs examens. Autrement dit, des étudiants en réussite sont susceptibles d'arrêter leurs études. Cela plaide en faveur du constat dressé par Orange et Bodin selon lequel l'importance des circulations entre cursus cassent la représentation tubulaire traditionnellement associée aux filières universitaires. Cela permet également de nuancer la théâtralisation du drame de l'échec à l'université et de l'abandon des études puisque la proportion d'étudiants quittant l'université avant l'obtention du diplôme préparé et ne se réinscrivant dans aucune formation l'année suivante est minime au regard de l'ensemble des inscrits $(5,1 \%)$. Cela confirme aussi l'intérêt de proposer une photographie du phénomène à un instant $\mathrm{T}$, là où d'ordinaire les instruments de mesure se fondent sur des suivis de cohorte d'une partie des étudiants. Or, l'importance donnée au phénomène varie nécessairement selon la population de référence retenue. Ramenée à l'ensemble de la population sortante (10 643 inscrits), les sorties anticipées représentent un quart des sorties (25,4\%), c'est-à-dire une part comparable aux données produites par l'enquête Génération du Céreq. Rapportée à l'ensemble des inscrits (sortants et non sortants), la part d'étudiants n'ayant pas obtenu le diplômé préparé et non réinscrits en formation devient « marginale ».

La part marginale du phénomène n'exonère pas d'analyse plus fine de l'abandon précoce des études supérieures. Cet abandon a des causes multiples. Outre le décès (10 observations), on en distingue quatre donnant lieu à plusieurs types d'étudiants : les « fantômes » inscrits mais non présents, les «persévérants » inscrits en second cycle, les « cumulards » inscrits en premier cycle mais déjà diplômés de l'enseignement supérieur, les « accrocheurs » inscrits en premier cycle non diplômés de l'enseignement supérieur. Parmi les 2710 « décrocheurs », 8 \% déclarent n'avoir jamais assisté à aucun cours. Parmi eux, $13 \%$ déclarent s'être inscrits pour les avantages financiers et sociaux 
du statut étudiant. Pour les autres, leur absence relève davantage d'un empêchement lié à des contraintes professionnelles, matérielles ou personnelles (Graphique 2).

Tableau 3 : Motifs exprimés par les étudiants n'ayant suivi aucun cours après leur inscription en 2015-2016 (en \%)

\begin{tabular}{|l|l|}
\hline Mon activité professionnelle ne me permettait pas de suivre la formation & 34,9 \\
\hline Une autre raison a modifié mon projet d'études & 22,9 \\
\hline J'ai été reçu·e dans une autre formation & 15,6 \\
\hline Je me suis inscrit·e pour bénéficier du statut étudiant & 13,3 \\
\hline La formation était trop éloignée de mon lieu de résidence & 7,3 \\
\hline Mon état de santé ne m'a pas permis de suivre la formation & 3,7 \\
\hline Les frais de la vie étudiante (logement, repas, etc.) étaient trop élevés & 1,4 \\
\hline L'état de santé d'une personne de mon entourage ne m'a pas permis de \\
suivre la formation & 0,9 \\
\hline Total & 100 \\
\hline
\end{tabular}

Source : Enquête ComUE, population de référence : 218 répondants). La question est : « Pour quelle raison principale n'êtes-vous jamais allé·e en cours?».

Lecture : parmi les étudiants n'ayant suivi aucun cours après leur inscription en 2015-2016, $35 \%$ justifient leur absence aux cours par une activité professionnelle menée en parallèle.

Dans les «sorties anticipées », on trouve aussi 1010 étudiants déjà titulaires d'un diplôme d'enseignement supérieur, soit plus d'un sortant sur trois. Parmi eux, 274 étaient pourtant inscrits en premier cycle (cursus DUT ou licence). Parmi les 274 sortants de premier cycle déjà détenteurs d'un titre, plus de la moitié déclare être 
diplômée d'un BTS ou d'un DUT, un quart est titulaire d'une licence générale ou professionnelle et $9 \%$ sont diplômés d'un niveau supérieur (master ou doctorat). Au total, parmi les 2710 «abandonnistes », seuls 1472 (soit 54\%) répondent à l'ensemble des critères d'une sortie sans diplôme : quitter l'enseignement supérieur sans n'y avoir obtenu aucun diplôme malgré une présence aux cours. Ces étudiants sortis non diplômés en 2015-2016 représentent 3,8\% de l'ensemble des inscrits en cursus DUT et licence la même année. Ce n'est donc pas cette proportion, somme toute faible, qui fait « problème » que le parcours des étudiants dans l'espace universitaire. Il s'avère que les parcours des étudiants en poursuite ou en arrêt des études varient en fonction d'une part des cursus et des filières, d'autre part des caractéristiques des étudiants. De ce point de vue, l'analyse des correspondances multiples construites à partir des plusieurs variables socio-démographiques, scolaires et universitaires permet de voir dès l'abord des différences importantes (graphique 3).

Graphique 3 : Analyse des correspondances multiples des parcours en 2016-2017 (inscrits 2015-2016) 


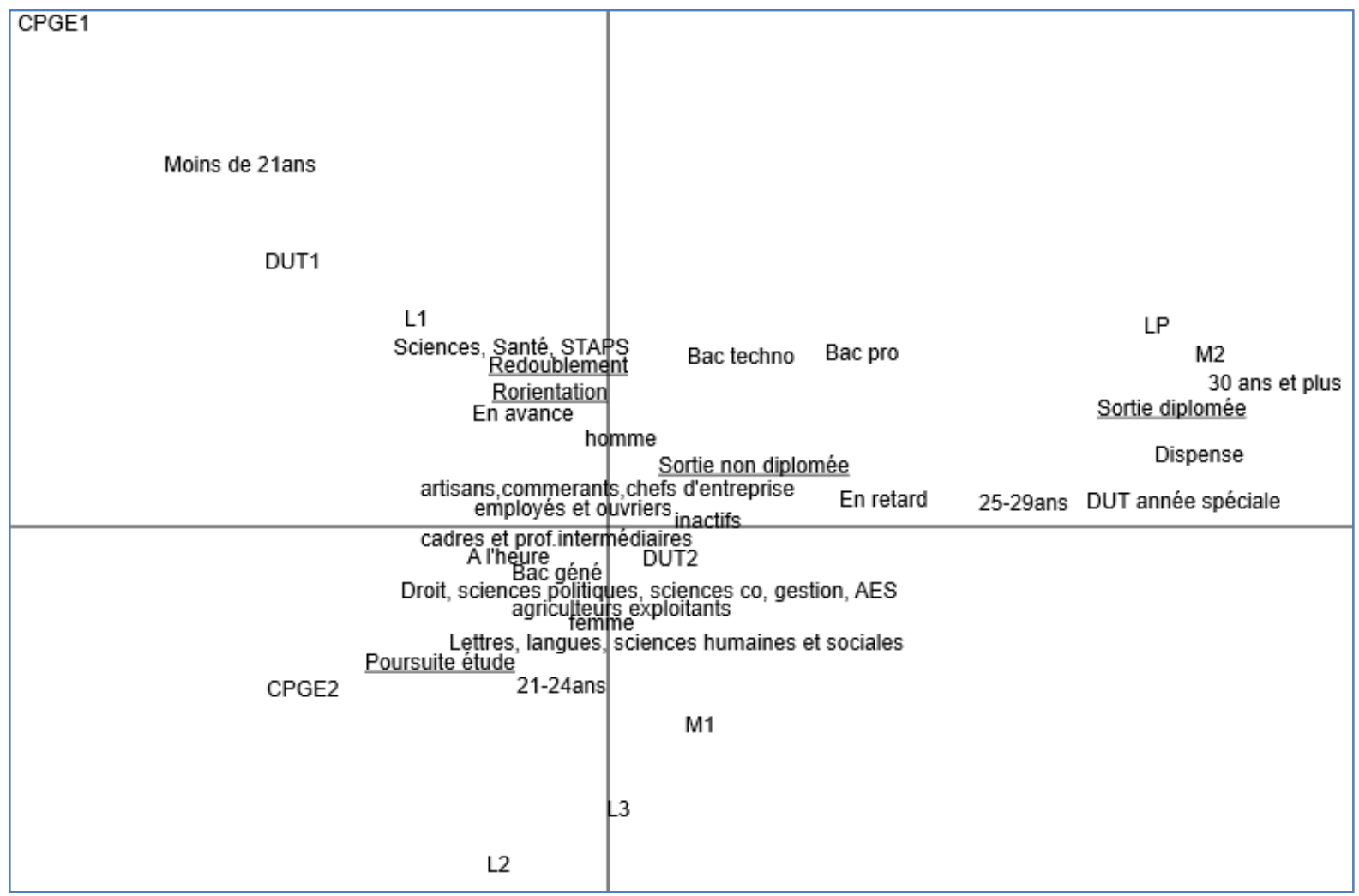

Ce plan factoriel, dont le premier axe (horizontal) apporte $15,20 \%$ de l'information et le deuxième axe (vertical) $8,1 \%$, livre plusieurs renseignements. Le premier est la présence d'un effet Guttman révélé par la lecture globale du plan factoriel. Cet effet est représenté par la trajectoire parabolique des niveaux d'études (depuis la CPGE1 aux M2 et LP). Il est dû à l'opposition sur l'axe horizontal des individus extrêmes alors que l'axe vertical oppose les individus moyens aux deux extrêmes.

S'il s'avère que cet effet est habituel dans le cas de variables temporelles (ici le niveau des études), il demeure intéressant dans l'étude des parcours d'études puisqu'il oppose sur l'axe horizontal la première année de licence et de CPGE aux dernières années d'études, tandis que l'axe vertical oppose les groupes de niveau d'études extrêmes (première et dernière année) aux groupes d'années intermédiaires. Ce faisant, certains points le long de la parabole méritent une attention particulière. Initialement assez importante, la proportion des étudiants les moins âgés qui se réorientent ou redoublent décroît à mesure que les étudiants sont plus âgés (21-24 ans). A cet âge, elle 
correspond aux étudiants en poursuite des études. La courbe croit à nouveau pour traduire l'importance des étudiants parmi les plus âgés qui sortent diplômés de l'enseignement supérieur. Par ailleurs, on note la place particulière des étudiants en CPGE de première année et qui sont parmi les étudiants les moins âgés. On suppose que cela est dû à l'excellence de leur parcours scolaire, au même titre d'ailleurs que les étudiants en DUT de première année. On les retrouve ensuite en poursuite des études la deuxième année, à l'instar des étudiants en L2, L3 et M1 des Sciences humaines et sociales, Droit, Sciences politiques et Sciences économiques.

Le deuxième renseignement concerne les redoublements et les réorientations qui d'une part interviennent plutôt en L1 dans les filières Sciences, Santé (Paces) et Staps, d'autre part sont plutôt le fait d'étudiants par le passé en réussite scolaire. Ayant obtenu leur baccalauréat avant l'heure, ce temps gagné est un atout pour rejouer un parcours universitaire sans accuser un retard sur l'agenda universitaire. Le troisième renseignement est la réussite en master $2^{\mathrm{e}}$ année et en licence professionnelle des étudiants plus âgés. On suppose ici qu'il s'agit plutôt d'étudiants étrangers majoritairement dispensés du baccalauréat ainsi que d'étudiants en reprise d'études. Le quatrième renseignement est le poids des conditions économiques et du passé scolaire sur la sortie sans diplôme. Celle-ci semble concerner plutôt des hommes, titulaires d'un baccalauréat professionnel ou technologique, qui ont redoublé durant leur scolarité, dont les parents sont d'une catégorie sociale défavorisées. De ce point de vue, cette information conforte les enquêtes sur l'échec à l'université.

Enfin, le graphique livre un cinquième élément : la distinction qui apparaît clairement entre les individus qui restent dans l'enseignement supérieur (poursuite d'étude, redoublement, réorientation) et les individus qui sortent (diplômés ou non). Les premiers sont les plus jeunes (moins de 21 ans ; 2124 ans), dans les premiers niveaux 
de licence ou DUT, qui sont «à l'heure » et titulaires d'un bac général, restent dans l'enseignement. Les seconds sont titulaires d'un bac technologique ou professionnel. Ils sont parmi les plus âgés et « en retard » sur leur parcours scolaire antérieur.

Plus largement, ces renseignements confirment que la sortie sans diplôme de l'enseignement supérieure varie selon les facteurs sociodémographiques et scolaires des étudiants ainsi que la filière et l'année d'étude. En conséquence, il importe d'affiner l'analyse en observant la part explicative de chaque variable sur le phénomène. À cette fin, un modèle de régression logistique permettant d'observer toute chose égale par ailleurs l'effet des facteurs sur la sortie sans diplôme a été construit par introduction de plusieurs variables dans le modèle (Tableau 4). Aux caractéristiques sociodémographiques (le sexe, l'âge, la PCS du parent de référence, et la nationalité) ont été ajoutés le type de bac, l'âge à l'obtention du bac, le niveau et la filière d'inscription au moment de la sortie.

Tableau 4 : Probabilité des étudiants inscrits en 2015-2016 d'être en sortie anticipée en 2017-2018

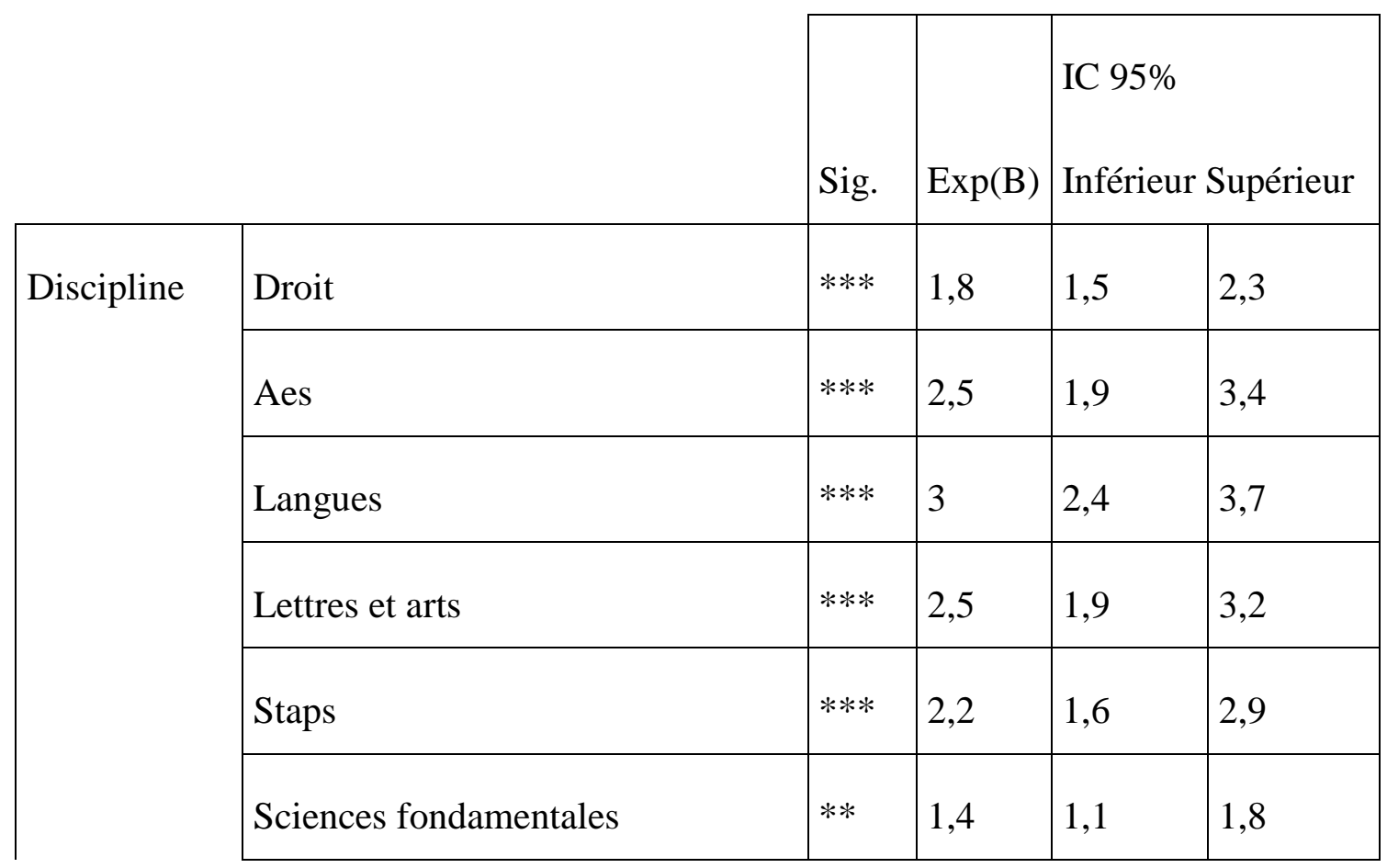


The Journal of Quality in Education (JoQiE) Vol.11, N¹7, May 2021

\begin{tabular}{|c|c|c|c|c|c|}
\hline & SHS & $* * *$ & 2,5 & 2,0 & 3,1 \\
\hline & Eco et gestion & $* * *$ & 2 & 1,5 & 2,5 \\
\hline & SVT & $* * *$ & 1,8 & 1,4 & 2,3 \\
\hline & Santé (réf.) & & & & \\
\hline \multirow[t]{8}{*}{ Niveau } & L1 & $* * *$ & 7,6 & 6,2 & 9,5 \\
\hline & L2 & $* * *$ & 2,5 & 2,0 & 3,1 \\
\hline & L3 & $* *$ & 1,3 & 1,0 & 1,7 \\
\hline & LP & $* *$ & 1,5 & 1,1 & 2,1 \\
\hline & DUT1 & $* * *$ & 2,8 & 2,0 & 3,9 \\
\hline & DUT2 & ns & & & \\
\hline & M1 & $* * *$ & 3,8 & 3,1 & 4,7 \\
\hline & M2 (réf.) & & & & \\
\hline \multirow[t]{5}{*}{ PCS } & Agriculteur exploitant & ns & & & \\
\hline & Artisan, commerçant, chef d'ent. & ns & & & \\
\hline & Cadre et PI & $* * *$ & 0,8 & 0,7 & 0,9 \\
\hline & Inactif & $\mathrm{ns}$ & & & \\
\hline & Employé Ouvrier (réf.) & & & & \\
\hline \multirow[t]{4}{*}{ Type de bac } & Bac général & $* * *$ & 0,3 & 0,3 & 0,4 \\
\hline & Bac techno & $* * *$ & 0,6 & 0,5 & 0,8 \\
\hline & Dispense & $* * *$ & 0,3 & 0,2 & 0,4 \\
\hline & Bac Pro (réf.) & & & & \\
\hline
\end{tabular}


The Journal of Quality in Education (JoQiE) Vol.11, N²17, May 2021

\begin{tabular}{|c|c|c|c|c|c|}
\hline \multirow[t]{4}{*}{ Age } & Moins de 21 (réf. & & & & \\
\hline & $21-24$ & $* * *$ & 2,0 & 1,8 & 2,3 \\
\hline & $25-29$ & $* * *$ & 4,6 & 3,8 & 5,6 \\
\hline & 30 et + & $* * *$ & 7,0 & 5,5 & 9,0 \\
\hline \multirow[t]{3}{*}{ Parcours } & En avance & ns & & & \\
\hline & A l'heure & $* * *$ & 0,8 & 0,8 & 0,9 \\
\hline & En retard (réf.) & & & & \\
\hline \multirow[t]{2}{*}{ Nationalité } & Français (réf.) & & & & \\
\hline & Étranger & $* * *$ & 0,7 & 0,6 & 0,9 \\
\hline \multirow[t]{2}{*}{ Sexe } & Femme (réf.) & & & & \\
\hline & Homme & $* * *$ & 1,2 & 1,1 & 1,3 \\
\hline
\end{tabular}

Seuils de significativité : ***<.000, $* *<.05$. Validité du test de Hosmer-

Lemeshow. $3 \%$ de résidus (positifs ou négatifs) supérieurs à 2 écarts-types, $0 \%$ de résidus supérieurs à 3 écarts-types. Indicateur de la qualité du modèle par la courbe ROC : supérieur à 0.7 .

Champ : Ensemble des inscrits 2015-2016 en licence, DUT et master, hors CPGE et l'année spéciale de DUT (50 129 observations valides). Source : Enquête sortants (ComUE d'Aquitaine)

Lecture : Un étudiant inscrit en Langues a 3 fois plus de risque d'être en sortie anticipée qu'un étudiant inscrit en Santé.

Trois résultats sont à souligner.

D’abord, le modèle confirme la faible probabilité d'être en sortie précoce des enfants dont le parent de référence est cadre ou profession intellectuelle. 
Comparativement aux enfants d'origine sociale défavorisée (c'est-à-dire des enfants d'employés ou d'ouvriers), l'origine sociale favorisée protège du risque de sortie précoce. En outre, le fait d'être titulaire d'un bac général comparativement à un bac professionnel protège d'une sortie précoce, tout comme le fait de n'avoir jamais redoublé. Somme toute, ces constats vont dans le sens des conclusions des recherches en France sur la meilleure réussite dans l'enseignement secondaire et l'enseignement supérieur selon l'environnement familial et la trajectoire scolaire.

Le deuxième résultat est l'effet de la nationalité sur la sortie précoce. On note que les étudiants étrangers, comparativement aux étudiants natifs, ont un risque de $30 \%$ moins élevé de quitter l'enseignement supérieur précocement. Ce résultat mériterait d'être approfondi car il va à rebours des conclusions des recherches en France qui mettent en évidence la moins bonne réussite scolaire des enfants d'immigrés à l'école que les enfants de natifs ainsi que la persistance de ce phénomène tout au long de la scolarité (Vallet et Caille, 1996 ; Brinbaum et Kieffer, 2009). Les recherches sont unanimes pour expliquer cet état de fait par le rôle prépondérant des caractéristiques sociales des familles immigrées. Sur ce plan, notre résultat oblige sinon à revoir ces conclusions, du moins à approfondir l'analyse des parcours des étudiants étrangers dans l'enseignement supérieur à l'aune de leur origine sociale ainsi que de l'origine géographique et du niveau d'éducation de leurs parents (Ichou, 2018). Il invite également à observer ces parcours à la lumière des obligations légales conditionnant les cursus de ces étudiants dans l'enseignement supérieur français (conditions de renouvellement des titres de séjour, législation récente relative aux frais différenciés de scolarité, etc.).

Le troisième résultat concerne la filière et le niveau des études. Le détail des odds ratios montre d'abord la surexposition des étudiants de L1 au risque de sortie 
anticipée. Comparativement aux étudiants inscrits en M2, le fait d'être en première année de licence multiplie environ par 8 le risque de sortie précoce. De ce point de vue, le consensus sur la lutte contre le « décrochage » à l'université et l'urgence de traiter le « problème » au niveau de la licence sont pertinents. Cependant l'attention portée sur la licence, en particulier la première année, ne doit pas occulter l'importance du risque de « décrochage » en première année de master. Il s'avère qu'après la première année de licence, la première année de master représente un risque élevé de sortie précoce. Celleci multiplie ce risque par 4 environ. Cela signifie que le traitement du « décrochage » n'est pas réductible à l'entrée à l'université, et qu'il s'agit d'un problème plus large d'accès aux diplômes universitaires. Autrement dit, les sorties anticipées de formation relèvent moins d'une spécificité du premier cycle universitaire que d'un effet de seuil indissociable de la temporalité d'études puisque le risque en licence diminue entre la première et la troisième année et qu'il remonte en première année de master.

Le phénomène de sortie précoce a donc un double étalement dans le temps. D’abord important la première année, l'odd ratio perdure les deux années suivantes puis reprend de la vigueur en master (tableau 4). Le graphique 4 complète ce constat en montrant que le moment du départ des études supérieures intervient toute l'année de L1et non pas durant les seuls premiers mois12. Les sortants de L1 au cours du premier semestre ne sont «que »19,4\%. Le taux passe à 22,2 \% en L2 puis à 15,3\% en L3. En

\footnotetext{
${ }^{12}$ Parmi l'ensemble des sortants sans diplôme, un peu plus de la moitié (50,8 \%) déclare avoir suivi la totalité de l'année universitaire avant d'interrompre une formation après les examens du second semestre tandis que les abandons en cours de premier semestre et avant les examens ne représentent «que »16,8\% (on comptę Gussi 12,9\% de sortants après les examens du premier semestre). En outre, la lecture par année donne des résultats différents.
} 
M1, il est de 11,7 \%. Les taux de sortie sans diplôme après les examens du second semestre sont de 41,4\% en L1, 48,7\% en L2, 51,4\% en L3 et 68,7\% en M1. Cela indique que la sortie sans diplôme est un processus qui s'étale de la licence jusqu'au master, et qu'elle intervient avant ou après les examens du premier et du second semestre. De sorte que la croyance selon laquelle un abandon massif interviendrait les premiers temps de l'entrée à l'université doit être revue à l'aune des sorties sans diplôme non suivies d'une réinscription qui surviennent davantage dans un temps long et de façon silencieuse, en particulier après les examens du second semestre.

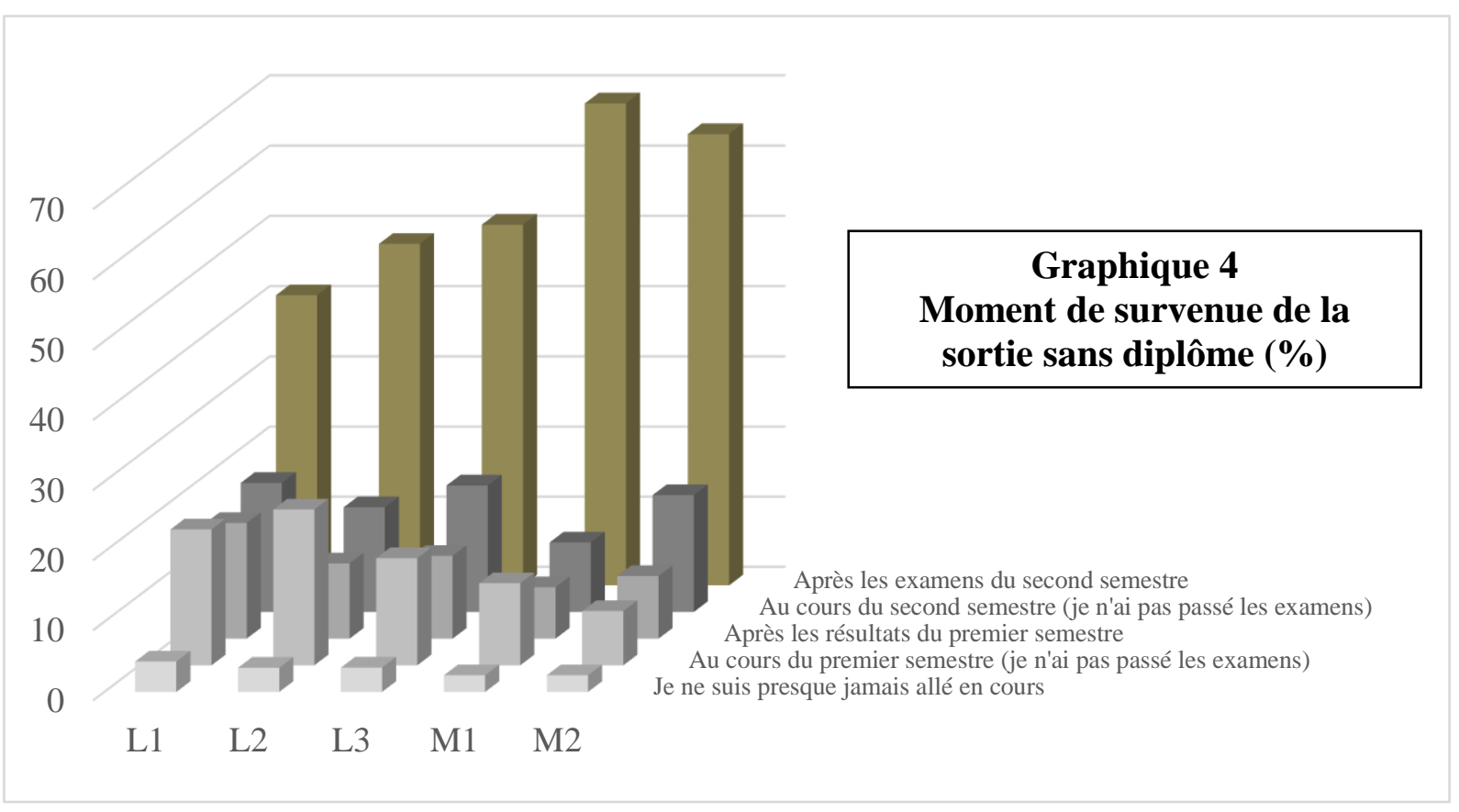

Champ : Ensemble des sortants 2015-2016 déclarant être allé au moins une fois en cours (hors CPGE). Source : Enquête sortants (ComUE d'Aquitaine)

Lecture : Parmi les étudiants non diplômés inscrit en L1 en 2015-2016 et non réinscrits en 2016-2017, 4,3\% ont interrompu leurs études avant d'avoir passé les examens de fin du premier semestre.

\section{Conclusion}

Issu d'une initiative européenne puis adaptée par les états nationaux, le «problème du 
décrochage universitaire » en France ne sort pas des cadres cognitifs de l'action publique qui ont permis son inscription à l'agenda politique (Hassenteufel, 2011). Nos résultats mettent en lumière l'écart entre la dramatisation du mal qui entoure le problème et les processus sociaux et temporels qui le fondent. De ce point de vue, deux résultats majeurs ressortent de notre enquête. Le premier est que les sorties sans diplôme de l'enseignement supérieur sont marginales au regard de l'ensemble des étudiants poursuivant un cursus universitaire. Le deuxième est qu'elles sont irréductibles au premier cycle universitaire. Tout spectaculaire que paraisse la désertion des amphithéâtres au cours du premier semestre universitaire, la majorité des sortants non réinscrits l'année suivante ont quitté l'enseignement supérieur soit après le premier semestre, soit après avoir effectué la totalité de l'année universitaire. Le risque de sortie sans diplôme n'est donc pas assimilable à un public d'étudiants égarés et désaffiliés. Or, le cycle de licence et plus spécifiquement la première année font l'objet d'une attention accrue des pouvoirs publics. Elle donne lieu à des dispositifs de prévention du décrochage qui s'adressent en priorité aux nouveaux entrants, ceux-là même qui sont justifiables d'un plan d'action en faveur de la réussite en licence.

Si nos résultats confirment une exposition de la première année universitaire au risque de sortie non diplômée, ils nuancent l'idée d'une spécificité propre aux nouveaux entrants puisque les étudiants inscrits en première année de master connaissent un risque de sortie anticipée. Par ailleurs, le processus varie dans le temps sans disparaître pour autant, ce qui va à l'encontre de l'image, construite par les politiques publiques et un pan de la recherche sociologique, du « décrochage » comme un «problème » spécifique à la première année de licence, en particulier les premiers mois suivant l'entrée à l'université lorsque le processus de désaffiliation battrait son plein (Coulon, ibid.). Les sorties sont loin de se concentrer sur les premiers mois suivants la rentrée 
universitaire. Cela amène à questionner davantage les caractéristiques des publics définis comme une cible prioritaire des dispositifs puisque les nouveaux entrants à l'université ne sont pas les seuls étudiants exposés au risque du « décrochage ».

Plus avant, nos résultats posent la question de la nature des interventions pédagogiques dans la construction des parcours d'études des étudiants. Il s'avère que le critère d'efficacité des dispositifs de lutte contre le « décrochage à l'université » se fonde sur la diminution du taux d'échec aux examens de première année. Or, l'échec peut conduire à une réorientation, à la suite de quoi il n'est plus un échec si elle est potentiellement bénéfique à l'étudiant. En outre, la sortie sans diplôme est un processus qui s’étale sur plusieurs années et qui varie en fonction de la discipline ainsi que des caractéristiques des étudiants et étudiantes. En conséquence, et comme le pointe Garcia (2009, 2010), le rôle des dispositifs est de garantir une offre de formation suffisamment large et des prestations suffisamment souples pour permettre les conditions de la réussite, quelles que soient l'année d'études et la filière.

En matière de rupture des parcours d'études supérieures et en dépit de l'ensemble des mesures prises pour éviter les ruptures, deux questions connexes restent centrales : celle des inégalités sociales et celle d'une inflation de dispositifs qui encourent le risque de rater leur cible.

\section{Bibliographie}

Annoot, E., 2012, «Les pratiques d'aide à la réussite: Du tutorat au plan licence », In Annoot E., La réussite à l'université: Du tutorat au plan licence, Louvain-laNeuve, De Boeck, pp. 111-138.

Annoot, E. (2001). Le tutorat ou «le temps suspendu ». Revue des sciences de l'éducation, 27 (2), pp.383-402.

Aschieri G., 2012, Réussir la démocratisation de l'enseignement supérieur : l'enjeu du premier cycle, Rapport du Conseil économique, social et environnemental. 
Beaud S., Millet M, « La réforme Macron de l'université », La Vie des idées, 20 février 2018.

Beaupère N., Boudesseul G. (dir.) (2009), Sortir sans diplôme de l'université.

Comprendre les parcours d'étudiants « décrocheurs », Observatoire national de la vie étudiante, Paris, La Documentation française.

Beaupère N., Boudesseul G., 2009, «Quitter l'université sans diplôme : quatre figures du décrochage étudiant », Bref, $\mathrm{n}^{\circ} 265$.

Bétant B., Foucault M. \& Peyroux C., 2010, Note relative à la mise en æuvre du plan pour la réussite en licence, Paris : Ministère de l'Enseignement supérieur et de la Recherche, n 2010-091.

Brinbaum, Y., Kieffer A., 2009, «Les scolarités des enfants d'immigrés de la sixième au baccalauréat : différenciation et polarisation des parcours », Population, 64, pp. 561-610.

Bodin, R. \& Millet, M., 2011, « La question de l'« abandon » et des inégalités dans les premiers cycles à l'université », Savoir/Agir, 17(3), 65-73.

Bodin R., Orange S., 2013, L'Université n'est pas en crise. Les transformations de l'enseignement supérieur : enjeux et idées reçues, Éditions du Croquant, coll. « Savoir/Agir », $213 \mathrm{p}$.

Calmand J., Ménard B., Mora V., 2015, « Faire des études supérieures, et après ? Enquête Génération 2010 - Interrogation 2013 », Note Emploi Formation, n 52, septembre, Céreq.

Castel R., 1995, Les métamorphoses de la question sociale. Une chronique du salariat, Paris, Fayard.

Céreq, 2014, «Le Portefeuille d'Expériences et de Compétences », Rapport d'évaluation, FEJ.

Cohen S., 2002 (1972), Folk Devils and Moral Panics. The Creation of the Mods and Rockers, New York, Routledge.

Cohen S., 2011, « Whose Side were we on? The Undeclared Politics of Moral Panic Theory », Crime Media Culture, 7, 3, pp. 237-243.

Commission européenne, 2012, «Repenser l'éducation - Investir dans les compétences pour de meilleurs résultats socio-économiques », Communication de la commission au parlement européen, au conseil, au comité économique et social européen et au comité des régions. 
Cohen, M.D., March, J.G. et Olsen, J.P., 1972, « A Garbage Can Model of Organizational Choice », Administrative Science Quarterly, 17(1), pp. 1-25.

Coulon A., 1997, Le métier d'étudiant : approche ethno méthodologique et institutionnelle de l'entrée dans la vie universitaire, Paris, PUF.

Crédoc, 2013, De l'université à l'entreprise, rapport d'évaluation, FEJ,

David S., Melnik-Olive E., 2014, «Le décrochage à l'université, un processus d'ajustement progressif ? », Formation Emploi, 2014/4, n 128, pp. 81-100.

Demuynck C., 2011, Réduire de moitié le décrochage universitaire, rapport à Monsieur le Premier ministre François Fillon, juin.

Demazière D., 2013, «Qu'est-ce que le travail d'accompagnement des chômeurs ? », Regards croisés sur l'économie, $\mathrm{n}^{\circ} 13$, pp. 137-150.

Dubet F., 1994, « Dimensions et figures de l'expérience étudiante dans l'université de masse », Revue française de sociologie, Volume 35, Numéro 4, pp. 511-532.

Durkheim É. 1887, «La Philosophie dans les universités allemandes », Revue international de l'enseignement, 13, pp. 313-38 et pp. 423-40 (Texte reproduit in Durkheim E., 1975, Textes, 3, Fonctions sociales et institutions, Paris, Éditions de Minuit, pp. 437-486).

Endrizzi L., 2010, « Réussir l'entrée dans l'enseignement supérieur », Dossier d'actualité de la VST, $\mathrm{n}^{\circ} 59$, décembre.

FEJ, Premiers enseignements des expérimentations en matière décrochage universitaire, note thématique, Octobre 2013

Felouzis G., 2001, La condition étudiante. Sociologie des étudiants et de l'université, Paris, PUF.

Galland O., Houzel G. (dir.), 2009, Les étudiants en France. Histoire et sociologie d'une nouvelle jeunesse, Rennes, PUR.

Garcia S, 2010, «Déscolarisation universitaire et rationalités étudiantes », Actes de la recherche en sciences sociales, 2010/3, $\mathrm{n}^{\circ} 183$, p. 48-57.

Garcia S., 2009, « Réformes de Bologne et économicisation de l'enseignement supérieur », Revue du MAUSS 2009/1 ( $\left.\mathrm{n}^{\circ} 33\right)$, p. 154-172.

Gautier C., 2015, «Le décrochage, le définir, le mesurer », Note OFIPE, n²2, Université Paris-Est Marne-la-Vallée.

Gury N., Moullet S., 2007, « L’insertion des non-diplômés de l'enseignement supérieur : réversibilités d'un échec et imbrication entre trajectoires scolaire et professionnelle », Relief, ${ }^{\circ} 22$, pp. 191-2010. 
Hassenteufel P., 2011, Sociologie politique : l'action publique, Paris, Armand Colin. Hetzel P., 2006, De l'université à l'emploi : rapport final de la Commission du débat national Université-Emploi, La Documentation française, Collection des rapports officiels, Octobre.

Ichou M., 2018, Les enfants d'immigrés à l'école. Inégalités scolaires, du primaire à l'enseignement supérieur, Paris, PUF.

Laval C., 2009, «Les nouvelles usines du savoir du capitalisme universitaire », Revue du MAUSS, 2009/1 (n³3), p 173-184

Lemaire S., 2005, « Que deviennent les bacheliers après leur baccalauréat ? », France, portrait social.

Livet P., 2012. « Normes sociales, normes morales, et modes de reconnaissance », Les Sciences de l'éducation - Pour l'Ère nouvelle, Vol. 45, pp. 51-66.

Mascherini S., Salvatore L., Meierkord A., Jungblut J.-M., 2012, Young people not in employment, education or training: Characteristics, costs and policy responses in Europe, Eurofound.

Ménard B., 2017, « Les sortants sans diplôme de l'enseignement supérieur en 2010 : tous décrocheurs ? Une analyse à l'aune de l'approche des capabilités ». In: Rendement éducatif, parcours et inégalités dans l'insertion des jeunes, Céreq, pp.129-148.

Michaut C., 2012, «Réussite, échec et abandon des études dans l'enseignement supérieur français : quarante ans de recherche » In Romainville M. (éd.), Réussite, échec et abandon dans l'enseignement supérieur, De Boeck Supérieur, pp. 53-68.

Michaut C., 2003, «L'efficacité des dispositifs d'aide aux étudiants dans les universités » In Recherche \& Formation, N43, « Entrer à l'université. Le Tutorat méthodologique », pp. 101-113.

Millet M., 2012, «L'« échec » des étudiants de premiers cycles dans l'enseignement supérieur en France. Retours sur une notion ambiguë et descriptions empiriques », In Romainville M. (éd.), Réussite, échec et abandon dans l'enseignement supérieur, Louvain-la-Neuve, De Boeck Supérieur, pp. 69-88.

Muller P., Leca J., Majone G., Thoenig J-C., Duran P., 1996, « Enjeux, controverses et tendances de l'analyse des politiques publiques », In: Revue française de science politique, $46^{\mathrm{e}}$ année, $\mathrm{n}^{\circ} 1$, pp. 96-133

Observatoire de la vie étudiante, 2016, Repères 2016. 
Pérennès L., Pinte G., 2012, « Retour d'expérience(s) sur un dispositif visant à réduire l'échec en premier cycle universitaire : le Cycle d'Orientation et de Consolidation », Questions Vives, Vol.6, n${ }^{\circ} 16$.

Perret, C. \& Morlaix, S., 2014, «Des effets du plan réussite en licence sur la sélection universitaire en première année de licence », Carrefours de l'éducation, 38(2), pp. 175-191.

Perret C., Berthaud J., Benoist S., 2013, « Essai de mesure de l'efficacité différenciée d'un plan « Réussite en licence » selon les acquis initiaux des étudiants », Revue française de pédagogie, 183, pp. 83-98.

Perret C., 2013, «Les choix des équipes pédagogiques pour la réussite des étudiants : un éclairage via la cartographie du plan réussite en licence de l'Université de Bourgogne », Revue internationale de pédagogie de l'enseignement supérieur, 29(2), pp. 1-19.

Romainville M., Michaut C., 2012, «Conclusion », In Romainville M. et al., Réussite, échec et abandon dans l'enseignement supérieur, Louvain-la-Neuve, De Boeck Supérieur, pp. 251-262.

Romainville, M. (2006). Introduction, In Rege Colet N. (éd.), La pratique enseignante en mutation à l'université, Louvain-la-Neuve, De Boeck Supérieur, pp. 7-13.

Romainville M., 2002, « Promouvoir la réussite à l'université », Table ronde organisée par Mme Françoise Dupuis Ministre de l'Enseignement supérieur, Bruxelles, le 22 mars 2002, La réussite à l'université, éclairages pédagogiques, Facultés universitaires de Namur.

Sarfati F., 2013, « Peut-on décrocher de l'université ? Retour sur la construction d'un problème social », Agora débat Jeunesse, $\mathrm{n}^{\circ}$ 63, pp. 7-21.

Schéma Régional de l'Enseignement Supérieur, de la Recherche et de l'Innovation, SRESRI Aquitaine, décembre 2012.

Tinto V., 1975. «Dropout from higher education: A theoretical synthesis of recent research », Review of Educational Research, vol. 45, $\mathrm{n}^{\circ}$ 1, pp. 89-125.

Touraine A., 1972. Université et société aux États-Unis, Paris, Le Seuil.

Vallet L.-A., Caille J.-P., 1996, « Niveau en français et en mathématiques des élèves étrangers ou issus de l'immigration », Économie et statistique, 293, pp. 137153. 
Van de Velde C., 2016, « «J'aimerais que quelqu'un m'attende quelque part » : Visages et expériences des «neet»», Le blog de la Chaire de recherche sur les inégalités sociales et parcours de vie, novembre.

Zaffran J., 2004, «Le temps des études universitaires : le cas des travailleurs sociaux », Formation Emploi. N.86, pp. 25-38.

Zaffran J., 2015, «Lutte contre le décrochage scolaire et jeux d'échelle : des acteurs publics aux parcours individuels », Revista Educação, Sociedade \& Culturas, ${ }^{\circ}$ 45, pp. 161-175. 